Oriani, R.A. and J.C. Fisher. Energetic Charged Particles Produced in the Gas Phase by Electrolysis. in Tenth International Conference on Cold Fusion. 2003. Cambridge, MA: LENR-CANR.org. This paper was presented at the 10th International Conference on Cold Fusion. It may be different from the version published by World Scientific, Inc (2003) in the official Proceedings of the conference.

\title{
ENERGETIC CHARGED PARTICLES PRODUCED IN THE GAS PHASE BY ELECTROLYSIS
}

\author{
R.A. Oriani* and J.C. Fisher** \\ *112 Amundson Hall, University of Minnesota \\ Minneapolis, MN 55455, U.S.A. \\ orian001@umn.edu \\ **600 Arbol Verde, Carpinteria, CA 93013, U.S.A.
}

\begin{abstract}
CR-39 plastic detector chips suspended in the vapor over the electrolytic solution during electrolysis record the tracks of highly energetic charged particles. The probability that the track densities found in these detector chips and the generally smaller track densities found in controls belong to a common population is $3 \times 10^{-10}$ by the Mann-Whitney statistical test. It is therefore concluded that a nuclear reaction that originates in the vapor phase can accompany electrolysis. Occasionally huge numbers of nuclear tracks are recorded by detector chips in the vapor over active electrolysis cells. One such experiment is analyzed in which two contiguous detector chips recorded approximately 40,000 tracks. Analysis of track orientations shows that the shower of charged particles originated in a compact source in the vapor between the chips at about $2 \mathrm{~mm}$ from one of the chips. A new type of nuclear reaction is indicated.
\end{abstract}

\section{INTRODUCTION}

The use of small pieces of CR-39 plastic immersed in the electrolyte has demonstrated that highly energetic charged particles generated during electrolysis produced nuclear tracks. ${ }^{(1,2)}$ This has been done with solutions of $\mathrm{Li}_{2} \mathrm{SO}_{4}$ in either $\mathrm{D}_{2} \mathrm{O}$ or $\mathrm{H}_{2} \mathrm{O}$ using either $\mathrm{Pd}$ or $\mathrm{Ni}$ as cathode material. Because chemical reactions fail by several orders of magnitude to produce charged particles of sufficient energy to produce tracks in CR-39, the generation of tracks during 
electrolysis provides unambiguous evidence that a nuclear reaction of some kind can accompany electrolysis. In the present work, guided by some considerations of a theory developed by Fisher, ${ }^{(3)}$ we investigate the use of CR-39 detector plastic suspended in the gases above the liquid solution during electrolysis.

\section{Experimental Aspects}

The experimental details involved in the present work are essentially the same as those described in a previous publication. (1) Small pieces of CR-39 detector, plastic purchased from the Fukuvi Chemical Industry Company of Japan, were etched in $6.5 \mathrm{~N} \mathrm{KOH}$ at $65-75^{\circ} \mathrm{C}$, photographed, and then suspended in the gas space of a simple electrolysis cell (Fig. 1). The gas space is surrounded by a heating coil to maintain a temperature of about $65^{\circ} \mathrm{C}$ in order to reduce condensation of moisture upon the suspended detector chips. A nickel disc set between the chips and the surface of the electrolyte serves to decrease carry-over of electrolyte solution mist to the chips during electrolysis. It also serves to block charged particles originating in the electrolyte from impinging upon the detector chips. A platinum wire spiral serves as the anode. The cathode is a plate of palladium or a sheet of nickel clamped between O-rings. The electrolyte is a solution of $0.023 \mathrm{~g} \mathrm{Li}_{2} \mathrm{SO}_{4}$ per $\mathrm{ml}$ of $\mathrm{H}_{2} \mathrm{O}$. Electrolysis at current densities between 0.1 and $0.4 \mathrm{~A} / \mathrm{cm}^{2}$ was carried out usually for three days, after which the detector chips were again etched and photographed at exactly the same areas as those before electrolysis. The number of energetic particle tracks produced during the electrolysis protocol is obtained by subtracting from the number found after electrolysis the number observed in the same areas before electrolysis. For each detector chip at least ten areas are counted and the counts are averaged to yield the mean numbers of new nuclear tracks per $0.008 \mathrm{~cm}^{2}$ of detector area. These numbers are compared with the numbers of tracks produced by radon during the preparation and handling of chips. For this purpose we expose detector chips to the vapor above the electrolyte solutions held in the electrolysis cell together with the electrodes. The control chips are treated in the 
same way and for the same length of time as the experimental chips except that they are not exposed to electrolysis.

\section{$\underline{\text { Results and Discussions }}$}

Table 1 presents the numbers of new tracks per $\mathrm{cm}^{2}$ found on 106 detector chip surfaces after electrolysis upon either Pd or Ni cathodes. The number density of new tracks found on 42 control chip surfaces are shown in Table 2. Because of the overlap at the lower ends of these data sets, a careful statistical analysis is required to assess the significance of the obvious difference between the two tabulations. We seek the probability, p, that the two data sets are simply portions of the same population. For this purpose we apply the Mann-Whitney test, ${ }^{(4)}$ a conservative analysis that does not depend upon whether or not a data set obeys a specific mathematical distribution. The result of the test is $\mathrm{p}=3 \times 10^{-10}$, so that there is only a vanishingly small probability that the data from experimental chips and the control chips constitute a single population. We therefore conclude that electrolysis can generate highly energetic charged particles by some kind of nuclear reaction that takes place in the gas phase. The values in Table 1 that lie in the range of values for the controls may have arisen because a nuclear reaction did not occur in those particular experiments, or because the chips were not located or oriented properly to receive the output of the nuclear reaction. The latter reasoning is realistic, since we have observed many instances when one side of a detector chip showed a large density of new nuclear tracks with the other side showing very few tracks. Evidently, the nuclear reaction does not occur uniformly in the gas phase. The occurrence of a nuclear reaction in the gas phase during electrolysis cannot be rationalized by any current theory except that developed by Fisher.(3)

\section{The Shower Phenomenon}

In addition to the results shown in Table 1, detector chips exposed in the gas phase in some experiments recorded very large numbers of new nuclear tracks after electrolysis. An 
example of what we have come to refer to as a shower is shown in Figure 2. We focus attention on one experiment with four detector chips suspended above the $\mathrm{H}_{2} \mathrm{O}$ solution of $\mathrm{Li}_{2} \mathrm{SO}_{4}$ with Pd as cathode material. Two of these chips developed track densities considerably above levels in the range of controls, but the other two showed enormous numbers of tracks on the sides facing each other and much smaller numbers on their rear sides.

Two hundred seventy areas of the densely pitted side of one of these chips were photographed and each of these photographs was subdivided into four equal-sized images. The etch pits on each image were counted, the total summing to 29,800 . We estimated that the portions of the chip unavailable for counting, because of the laser-inscribed numerals on the chip and because of the suspending wire, contained another 3540 etch pits, bringing the total number to about 33,300 charged particles that impinged upon the areas covered by the 1044 images. A contour plot of the number density of etch pits is shown in Figure 3. There is a marked maximum of more than 110 pits per image (i.e., $176,000 / \mathrm{cm}^{2}$ ) near the lower left portion of the chip, with the number density decreasing to background level at the dashed contour line for 3 pits per image. This background level is the number density typically found in chips exposed to the vapor in experiments in which a massive shower of energetic particles did not take place. This contour line is therefore taken as the boundary beyond which the shower of particles did not produce tracks. The gradient of pit density displayed in Figure 3 suggests that the contour line for 3 pits per image lies just beyond the lower left corner of the chip.

Figure 4 shows the etch pits on one small area of the chip. The etch pits vary from perfectly circular to sharply semi-elliptical. The circular pits were caused by charged particles impinging nearly perpendicularly upon the detector chip. Particles impinging at smaller angles with respect to the surface produced cones whose eccentricity increases as the angle of approach decreases. The axis of the cone is parallel to the line of travel of the impinging particle, and the vertex points in the direction of travel. The directions of the tracks were determined in nine areas around the perimeter about the more densely pitted portion of the chip. Each pit with a determined orientation was assigned a unit vector in the direction of the track as seen in its 
projection on the chip surface. In each of the nine areas the unit vectors were summed, thereby obtaining the average direction of the tracks in that area. The cosine of the angle between each unit vector and the mean direction of the tracks in each area was determined and the mean cosine was calculated. The mean cosine measures the degree of mutual alignment of the unit vectors; a mean cosine near unity signifies a nearly parallel alignment, and one near zero signifies a tendency of the unit vectors to point in opposite directions. The arrows in Figure 3 indicate the mean orientation of the tracks in each area, and the lengths of the arrows are proportional to the mean cosines, i.e., the degree of alignment, of the track projections along these directions. The central area of highest-density pitting has predominantly circular tracks.

It is clear from Figure 3 that the shower of charged particles that created the tracks originated somewhere in the vapor above the lower left corner of the chip. One can also infer that the source of the particles was not stationary during the duration of the burst because of the absence of circular symmetry in the density distribution. A more quantitative estimate of the location of the source of charged particles may be obtained by considering the etching process. Etching causes overall recession of the surface of the chip at a rate smaller than the advance along the trail of damage due to the impinging charged particle. Hence etching leaves a pit that marks the trail, but not if the angle of the trail with respect to the surface is small. The reason for this is that any spot on a shallow enough damage trail is reached by the receding surface before it can be reached by (the faster) etching along the trail (Fig. 5A). Thus, there is a critical angle of particle impingement smaller than which etching does not leave a pit to mark the damage trail. The critical angle is in fact equal to the half-angle of the cone-shaped pit. Careful measurements of the conical pits established the critical angle at about 36 degrees. We therefore deduce that all particles were generated below sloping surfaces that rise from the 3 pit per image contour line at an angle of about 36 degrees. The intersection of these surfaces occurs at approximately 2 $\mathrm{mm}$ from the chip surface (Fig. 5B) near the region of maximum track density and at about $5 \mathrm{~mm}$ near the upper edge of the chip. This suggests that a fairly compact source of energetic particles moved away from the chip surface as it rose toward and past the support hole. 
From Fig. 3 one can see that if the detector chip had been larger, a larger area of high density of tracks would have been recorded. We estimate that about 50,000 pits would have been recorded if the chip had been sufficiently large. Furthermore, considering that particle trajectories do not produce pits making angles less than 36 degrees with the surface, we estimate that the burst produced about 250,000 charged particles in the complete $4 \pi$ steradians of solid angle.

As pointed out above, this chip faced another chip about $8 \mathrm{~mm}$ distant, both chips having been hung on hooks on opposite sides of the supporting rod of about $2 \mathrm{~mm}$ diameter. One might expect that a shower of particles originating near one chip would also have affected the other; indeed this is the case. Fig. 6 shows a contour plot of the number density of etch pits on the facing surface of the second chip, and the arrows provide measures of the degree of orientation of the particle tracks. A careful analysis ${ }^{(5)}$ indicates that the source of energetic particles moved upward from the lower towards the upper edge of this second chip, in qualitative agreement with the deduction based on the observations on the first chip. The number density of pits on the second chip is smaller than that on the first chip because of the greater distance between the second chip and the source of particles ( $6 \mathrm{~mm}$ vs. $2 \mathrm{~mm}$ at the initial position of the source).

\section{CONCLUSIONS}

We have demonstrated that a nuclear reaction can accompany electrolysis of a $\mathrm{Li}_{2} \mathrm{SO}_{4}$ solution in either $\mathrm{H}_{2} \mathrm{O}$ or $\mathrm{D}_{2} \mathrm{O}$ upon either $\mathrm{Pd}$ or $\mathrm{Ni}$. Furthermore, reaction can take place in the gas phase above the surface of the electrolyte, and it can occur in spatially localized bursts that last a few seconds. Our experiments furnish compelling evidence for low-temperature nuclear reactions of an entirely new kind, consistent only with the neutron isotope theory.(3) 


\section{REFERENCES}

1. R.A. Oriani and J.C. Fisher, Jpn. J. Appl. Phys. 41, 6180 (2003) and 42, 1498 (2003).

2. R.A. Oriani and J.C. Fisher, Trans. Am. Nuc. Soc. 88, 640 (2003).

3. J.C. Fisher, Proc. 10th Int. Conf. Cold Fusion (2003).

4. F. Mosteller and R.E.K. Rourke, "Sturdy Statistics: Nonparametric Order Statistics," Addison-Wesley Pub. Co., Reading, MA (1975).

5. R.A. Oriani and J.C. Fisher, submitted to SCIENCE. 
Table 1. Vapor-Exposed Detector Chips for $\mathrm{Pd} / \mathrm{H}_{2} \mathrm{O} / \mathrm{Li}_{2} \mathrm{SO}_{4}$, and $\mathrm{Ni} / \mathrm{H}_{2} \mathrm{O} / \mathrm{Li}_{2} \mathrm{SO}_{4}$

\begin{tabular}{|c|c|c|c|}
\hline \multicolumn{4}{|c|}{ Tracks $/ \mathrm{cm}^{2}$} \\
\hline 39 & 260 & 494 & $1105^{*}$ \\
\hline 65 & 260 & 507 & $1105^{*}$ \\
\hline 65 & 273 & $572 *$ & 1118 \\
\hline $65^{*}$ & 273 & 572 & $1183^{*}$ \\
\hline 78 & $273 *$ & 572 & 1209 \\
\hline 91 & 286 & 585 & 1274 \\
\hline 104 & 299 & 585 & $1456^{*}$ \\
\hline 104 & 312 & 611 & $1534 *$ \\
\hline 104 & 312 & $624 *$ & 1599 \\
\hline $117^{*}$ & 312 & 624 & $1690 *$ \\
\hline $117^{*}$ & 338 & $676^{*}$ & $1820 *$ \\
\hline 130 & 338 & $689 *$ & 1820 \\
\hline 130 & 351 & $689 *$ & $1846^{*}$ \\
\hline $143^{*}$ & 351 & 715 & $1898^{*}$ \\
\hline 143 & $351 *$ & $780 *$ & $2002 *$ \\
\hline 143 & $377 *$ & $832 *$ & $2002 *$ \\
\hline 143 & 390 & 832 & $2184^{*}$ \\
\hline 143 & 390 & 858 & $2535^{*}$ \\
\hline $156^{*}$ & 416 & $871 *$ & 2626 \\
\hline 156 & $429 *$ & $949 *$ & 2990 \\
\hline $208^{*}$ & 429 & 949 & $3060 *$ \\
\hline $234^{*}$ & 429 & 975 & $3107^{*}$ \\
\hline 234 & $442 *$ & 988 & $3770 *$ \\
\hline 247 & 455 & $1014^{*}$ & $3848 *$ \\
\hline 247 & $468 *$ & 1053 & 10,400 \\
\hline 260 & 494 & 1079 & \\
\hline 260 & 494 & 1092 & \\
\hline
\end{tabular}

*Ni used for cathode. All others Pd. 
Table 2. Control Chips

\begin{tabular}{cc}
\hline \multicolumn{2}{c}{ Tracks/cm } \\
\hline 38 & 150 \\
62 & 150 \\
62 & 150 \\
62 & 162 \\
75 & 175 \\
75 & 175 \\
88 & 188 \\
100 & 188 \\
112 & 200 \\
112 & 200 \\
112 & 200 \\
112 & 225 \\
125 & 238 \\
138 & 238 \\
150 & 262 \\
150 & 350 \\
\hline
\end{tabular}




\section{FIGURES}

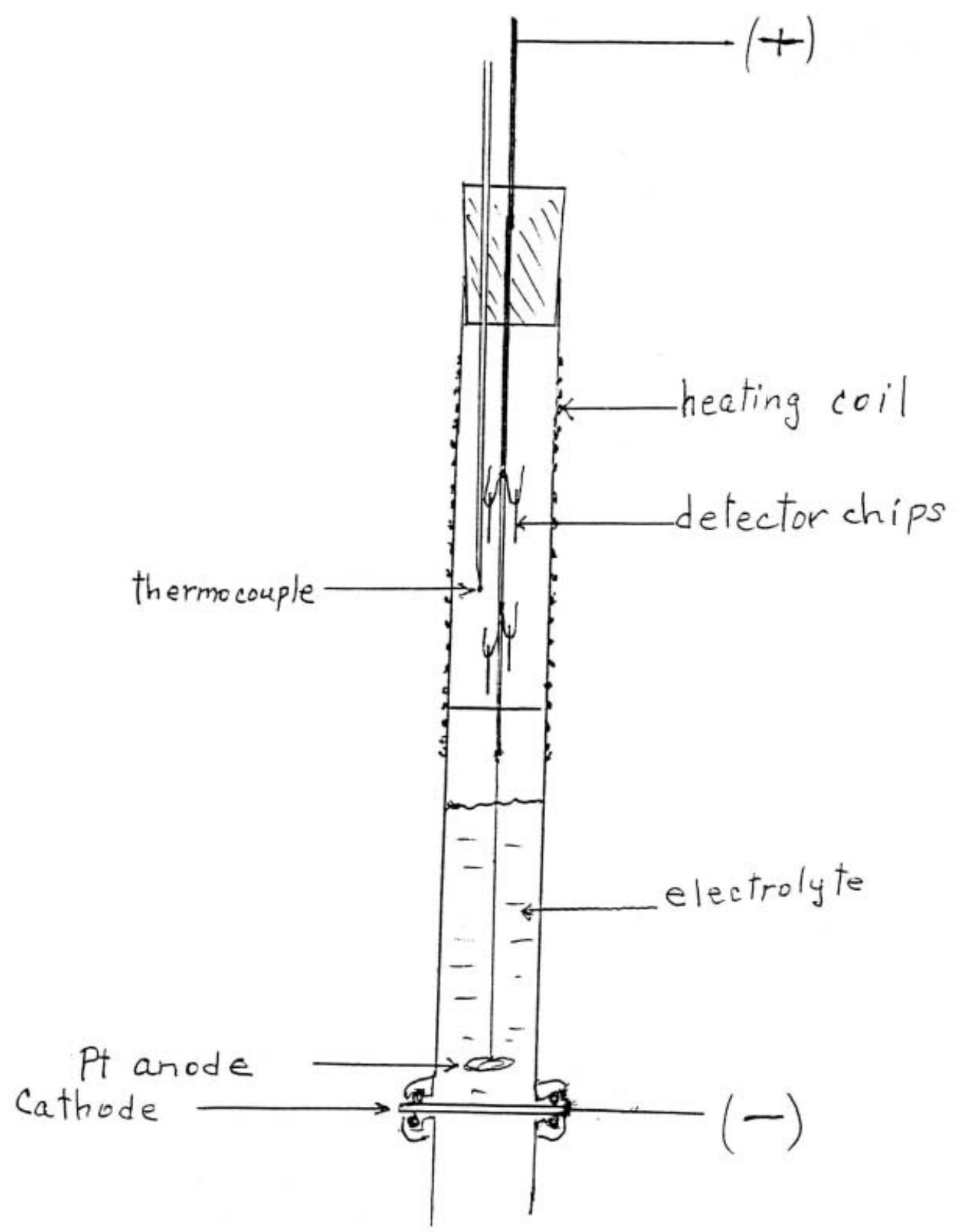

Figure 1. Schematic diagram of electrolysis cell. 

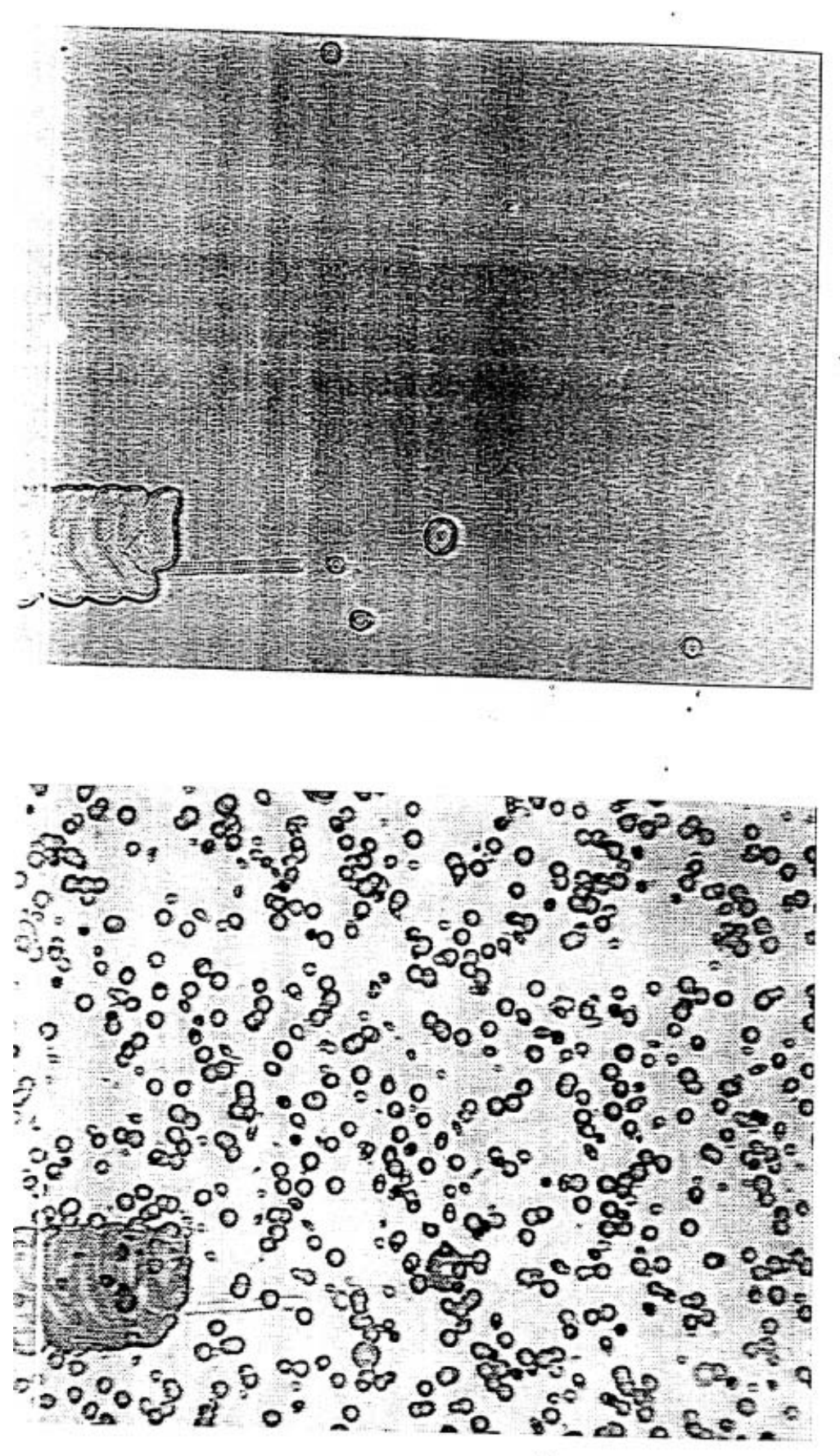

Figure 2. (A): CR-39 plastic detector showing radon-produced nuclear tracks in chip before exposure to electrolysis. Digital image processed by the Find Edges program, original magnification 100X. Feature in lower left corner of figure is a portion of a scratch. (B): The same area after exposure of the chip to electrolysis. 


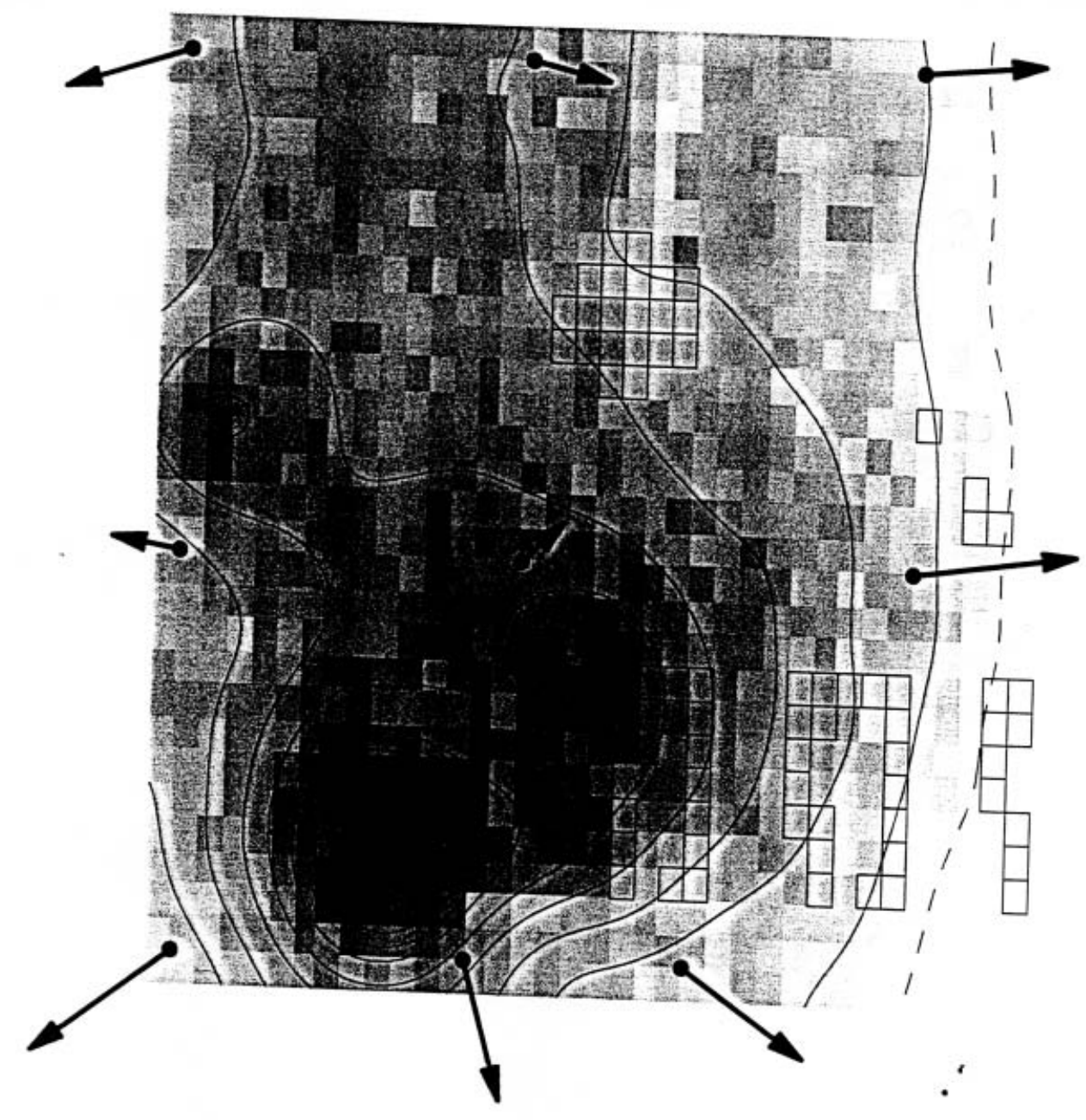

Figure 3. Distribution of nuclear tracks in a CR-39 plastic detector chip hung in the gas phase over the electrolyte during electrolysis. The approximately $8 \mathrm{~mm}$ square surface was photographed in 1044 images and the density of tracks per image is shown by smoothed contours spaced at 10 tracks/image. The contours range from 110 tracks/image near the peak to 3 along the right side of the figure. The arrows indicate the mean directions of the tracks at their locations, and their lengths are proportional to the degree of alignment of the tracks (see text) as measured by the mean cosines. Clockwise beginning with the arrow at the lower left corner, the values of the mean cosines and (in parentheses) the numbers of tracks from which they were determined are: 0.956(35), 0.387(43), 0.726(30), 0.453(49), 0.686(20), $0.925(30), 0.838(20), 0.798(72)$; for the central arrow they are $0.331(58)$. 


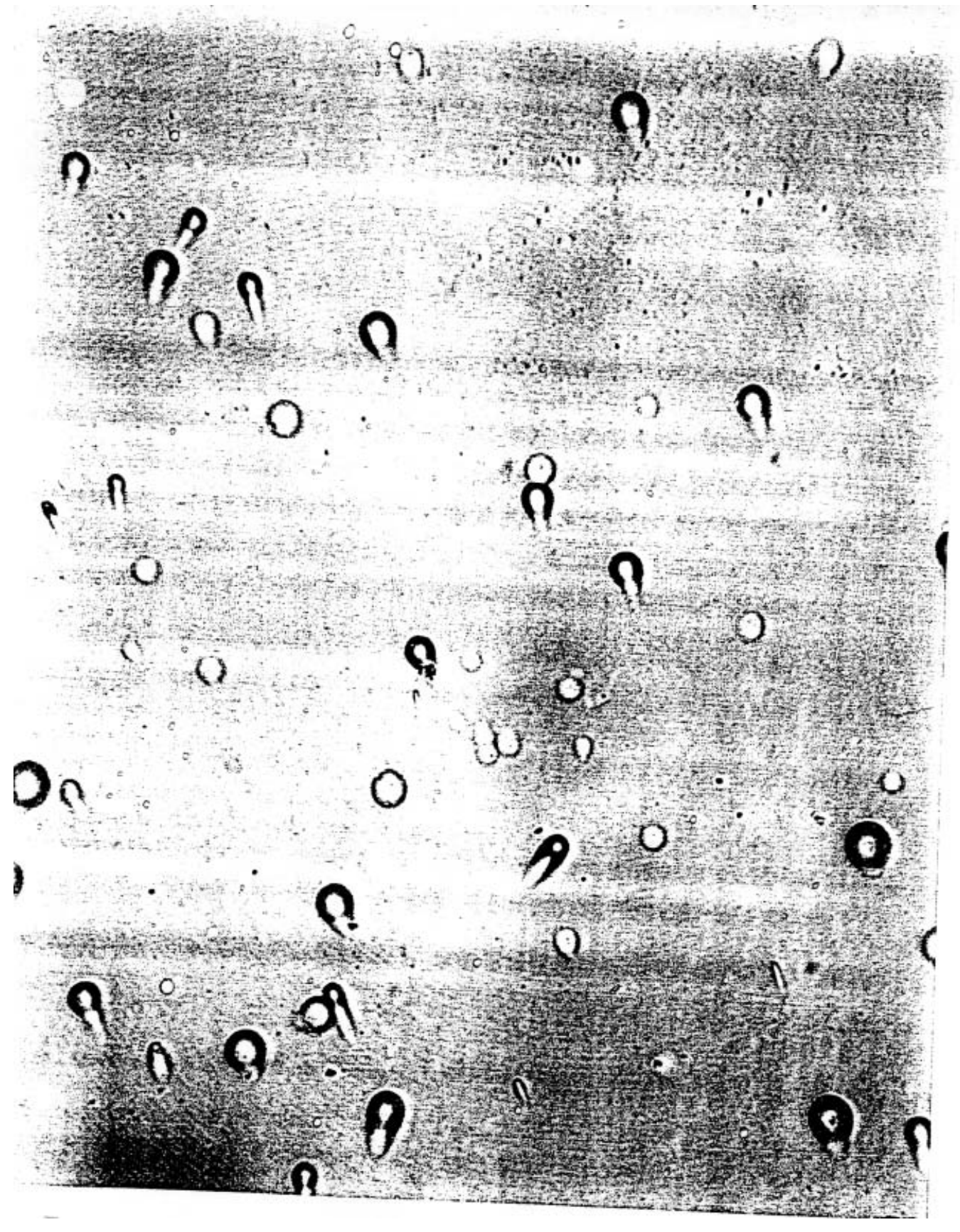

Figure 4. Image of nuclear tracks showing variety of shapes that depend on the angle of impingement of the charged particle. This image was not processed by the Find Edges program. 


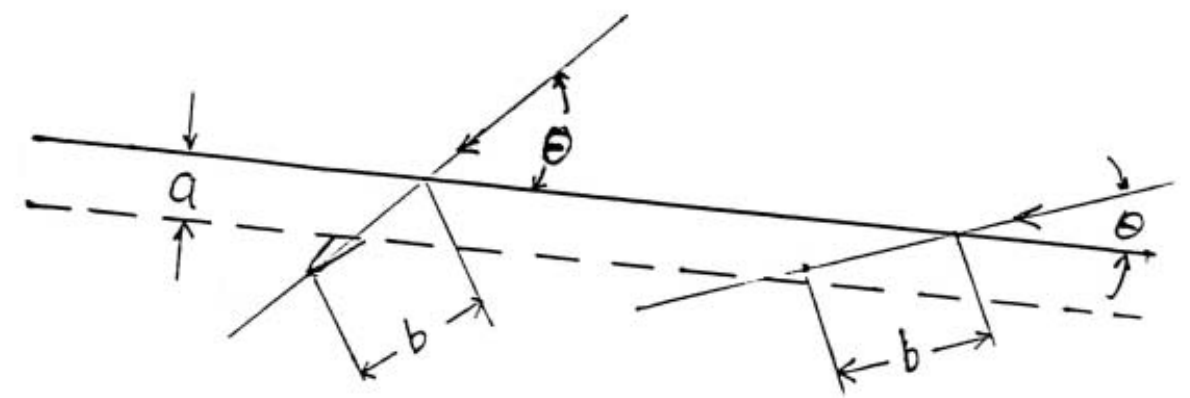

A.

$*$

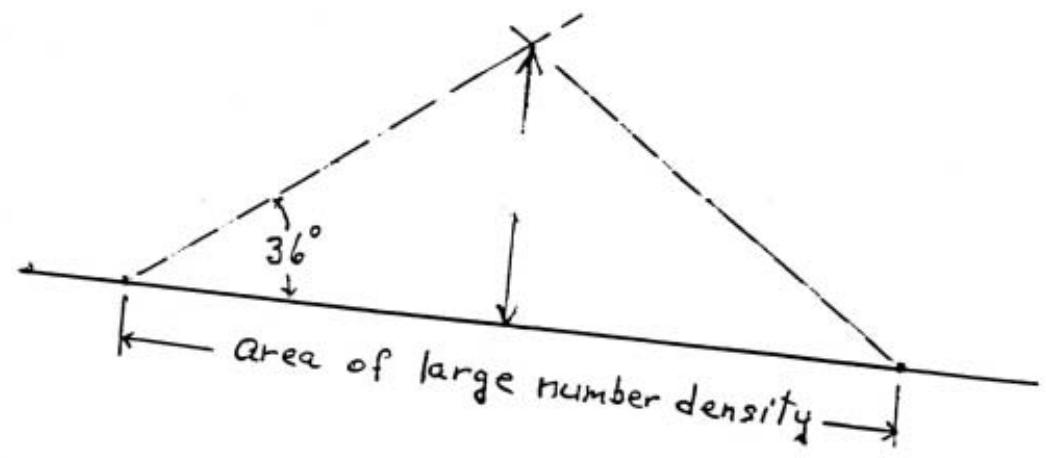

$B$

Figure 5. (A): Schematic diagram showing that etching will not reveal the track of a nuclear particle of its trajectory makes a small enough angle of impingement, $\theta$, with the surface. In unit time of etching the surface of the plastic recedes by a distance a, while etching proceeds along the particle's path by a distance $b$, where $b>a$. (B) Construction used to establish the approximate distance from the chip surface at which the shower of nuclear particles originated. 


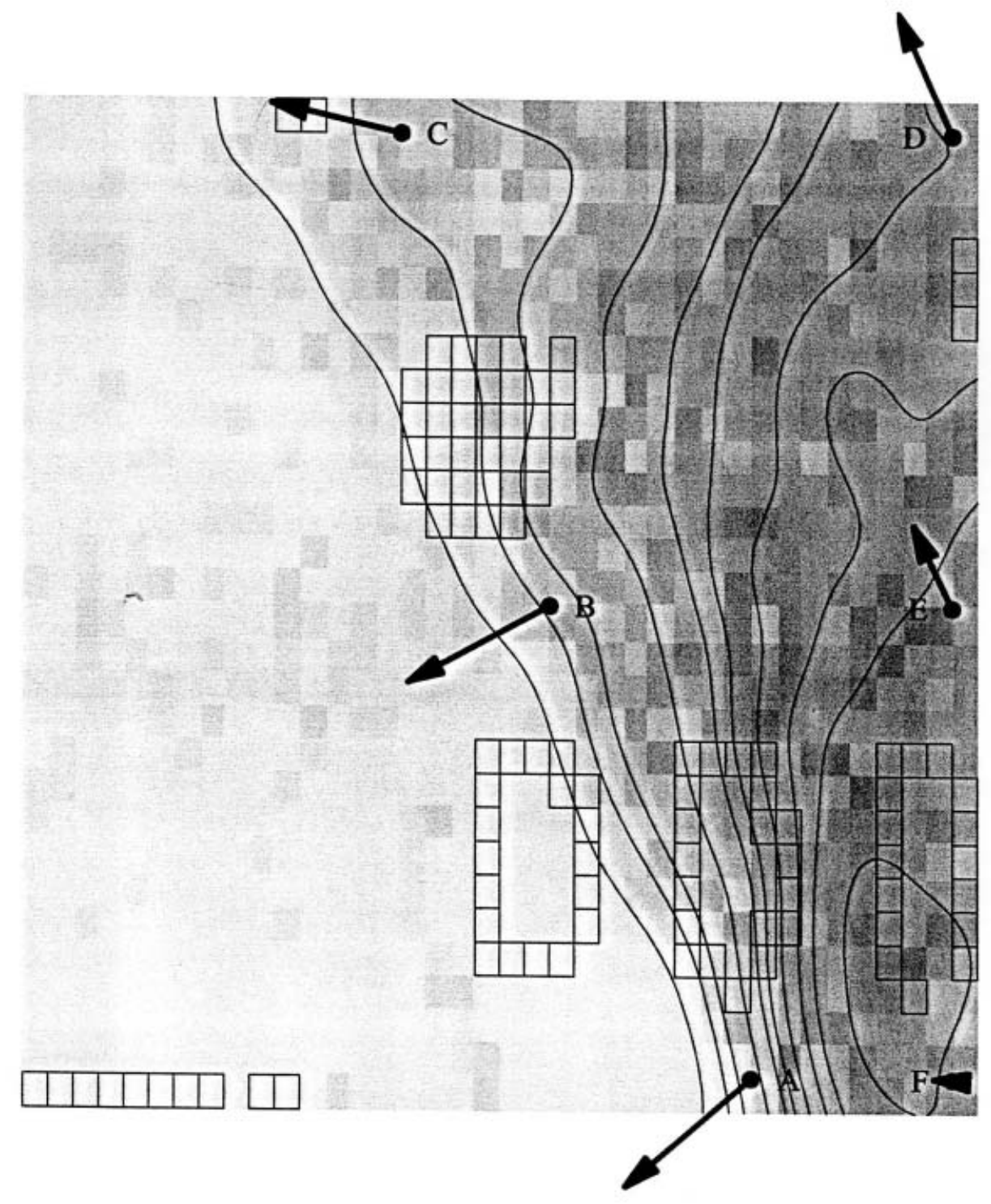

Figure 6. Contour plot of nuclear tracks produced on the companion detector chip about 8 $\mathrm{mm}$ away from the first chip. The smoothed contour lines are spaced at 3 pits/image and range from 3 pits/image on the left of the figure to 30 pits/image at the peak. 